\title{
$216 \mathrm{MHz}$ repetition rate passively mode-locked electrically-pumped VECSEL
}

\author{
A. Alhazime, M. Butkus, C. J. Hamilton, E. U. Rafailov
}

A. Alhazime, M. Butkus, C. J. Hamilton, E. U. Rafailov, "216 MHz repetition rate passively mode-locked electrically-pumped VECSEL," Proc. SPIE 8966, Vertical External Cavity Surface Emitting Lasers (VECSELs) IV, 89660K (3 March 2014); doi: 10.1117/12.2039275

SPIE. Event: SPIE LASE, 2014, San Francisco, California, United States 


\title{
$216 \mathrm{MHz}$ repetition rate passively mode-locked electrically pumped VECSEL
}

\author{
A.Alhazime ${ }^{\mathrm{a}}$, M. Butkus ${ }^{\mathrm{a}}$, C.J. Hamilton ${ }^{\mathrm{b}}$, E. U. Rafailov*a \\ ${ }^{a}$ Photonics and Nanoscience Group, School of Engineering, Physics and Mathematics, University of \\ Dundee, Dundee, DD1 4HN, UK; \\ ${ }^{\mathrm{b}} \mathrm{M}$ Squared Lasers Ltd, 1 Kelvin Campus, West of Scotland Science Park, Glasgow, G20 0SP, \\ United Kingdom
}

\begin{abstract}
Electrically pumped vertical external cavity surface emitting laser is passively mode-locked at record-low repetition rate of $216 \mathrm{MHz}$ demonstrating potential peak power scalability. A quantum dot saturable absorber is used to achieve stable operation.
\end{abstract}

Keywords: Electrically pumped VECSEL, SESAM, quantum dots, mode-locking.

\section{INTRODUCTION}

The concept of external cavity surface emitting semiconductor laser was successfully employed by optically pumped vertical external cavity surface emitting lasers (OP-VECSELs) also known as semiconductor disk lasers (SDLs). It allowed significant power scaling in both continuous wave and mode-locked regimes ${ }^{1-2}$. Lowest repetition rate demonstrated in mode-locked OP-VECSELs was $86 \mathrm{MHz}$ and $200 \mathrm{MHz}$ was demonstrated in pulsed SESAM-free configuration $^{3-4}$. OP-VECSELs were used for a number of applications, including non-linear imaging techniques in biophotonics ${ }^{5}$. Meanwhile electrically pumped vertical external cavity surface emitting lasers (EP-VECSELs) were envisioned to further reduce size footprint and increase efficiency keeping the flexibility of their optically pumped counterparts for lower power applications ${ }^{6}$. To date the output power from mode-locked EP-VECSEL was mainly constrained by limited contact aperture size and achievable beam quality. Meanwhile achievable pulse duration was defined by narrow optical bandwidth due to the semiconductor micro-cavity formed by two Bragg reflectors ${ }^{7}$. To date mode-locked EP-VECSELs with average output power up to $40 \mathrm{~mW}$ and pulses as short as 14.8 ps were demonstrated ${ }^{8-9}$. In this letter we focus on different parameter and report a significant reduction of laser repetition rate with subsequent increase of peak power. Quantum dot based semiconductor saturable absorber mirror (QD-SESAM) was used to modelock the laser allowing low pulse fluence and relieved cavity design. Average output power of $8 \mathrm{~mW}$ during the modelocked operation was achieved at a repetition rate of $216 \mathrm{MHz}$, with the pulse duration of $24 \mathrm{ps}$ at emission wavelength of $980 \mathrm{~nm}$. Subsequent optimization of all parameters will enable significant peak power scaling with values up to tens of watts.

\section{SAMPLES AND EXPERIMENTAL SETUP}

EP-VECSEL used in our work was produced by Novalux, US and had both its optical mode and their wavelength controlled by an extended compound optical cavity. The active region of the VECSEL was composed of several strain compensated InGaAs/GaAsP MQWs, grown by organometallic vapor deposition atop an n-type multilayer GaAs/AlGaAs Bragg reflector (DBR, R 0.7) and $n-G a A s$ substrate. A high reflectivity p-type DBR completed the epitaxial structure that was bonded through a dielectric layer with an aperture providing electrical contact to a heatsink. The n-contact defined an optical aperture within the device to help stabilise the output into a TEM00 mode. The n-DBR was to partly offset finite absorption losses for light travelling through the n-GaAs substrate, as well as to stabilise the frequency output of the VECSEL ${ }^{10}$.

*Contact author:e.u.rafailov@dundee.ac.uk 
A semiconductor saturable absorber mirror (SESAM) designed for $980 \mathrm{~nm}$ was grown by Molecular Beam Epitaxy (MBE). The absorbing section contained 2 layers of InGaAs quantum dots (QDs) sandwiched between GaAs barriers. The absorbing structure was resonant and grown on top of a DBR which had 28 pairs of $1 / 4$ lambda thick GaAs/AlGaAs layers. It had peak absorption at $967 \mathrm{~nm}$. QD SESAMs were previously successfully used to mode-lock solid state, fiber lasers and OP-VECSELs ${ }^{11-12}$. QD-SESAMs typically feature lower saturation fluence, faster recovery times and lower loss as compared to QW absorbers ${ }^{13}$. Luminescence spectra of both EP-VECSEL and SESAM are shown in Fig. 1.

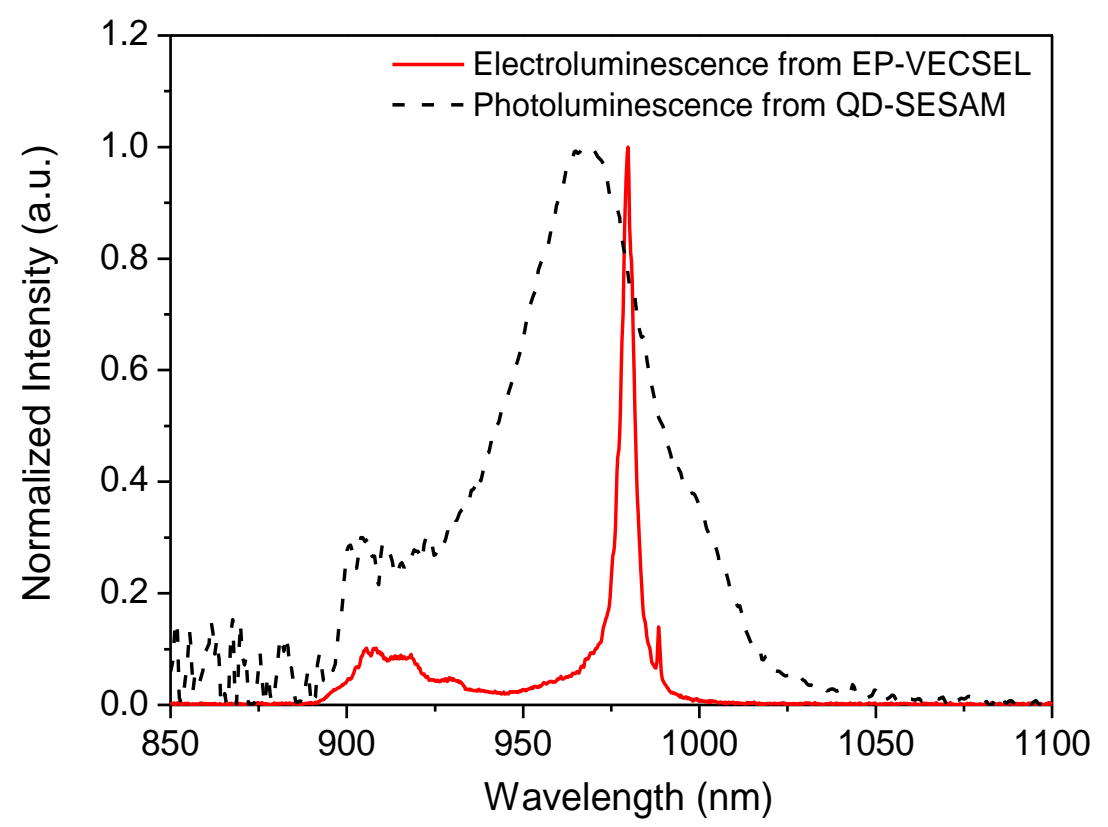

Figure 1. Red line shows electroluminescence measured from EP-VECSEL. Black line indicates the photoluminescence spectrum measured from top of the QD-SESAM.

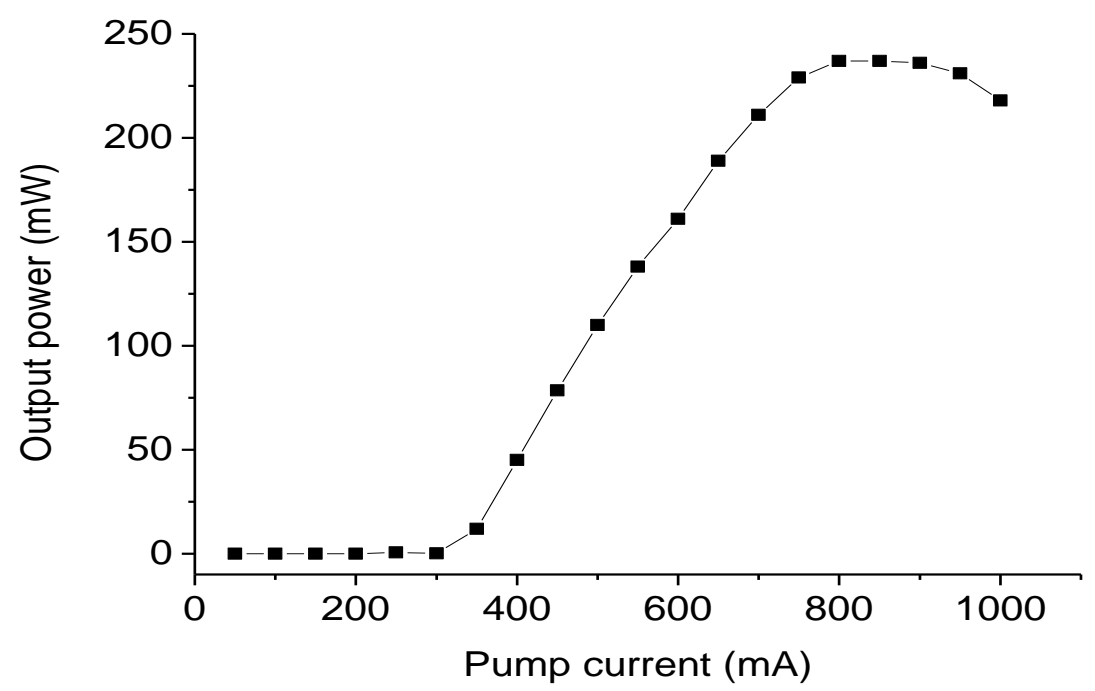

Figure 2. Current-light characteristics of EP-VECSEL operated in CW regime with $10 \%$ output coupler. 
The EP-VECSEL was first tested in CW regime. For it, a straight two-mirror cavity was formed using RoC $=-75 \mathrm{~mm}$ output coupler with $10 \%$ transmission at $980 \mathrm{~nm}$. In this configuration, output power up to $218 \mathrm{~mW}$ was achieved under pump current of $850 \mathrm{~mA}$. Current-output power characteristics curve of the device is shown in Fig. 2. For mode-locking experiments, a multi-folded cavity was built using plano-concave and plane mirrors. An output coupler with a RoC = $100 \mathrm{~mm}$ and $10 \%$ transmission formed one end of the cavity whilst the SESAM closed the other end. The gain and SESAM were mounted on temperature-controlled copper mounts. The temperature of QD-SESAM was set to $25^{\circ} \mathrm{C}$ and the QW gain structure was at $15^{\circ} \mathrm{C}$ for most stable operation. Mode spot sizes on gain and SESAM were set to $75 \mu \mathrm{m}$ in radius. Schematic drawing of experimental laser cavity is shown in Fig.3.

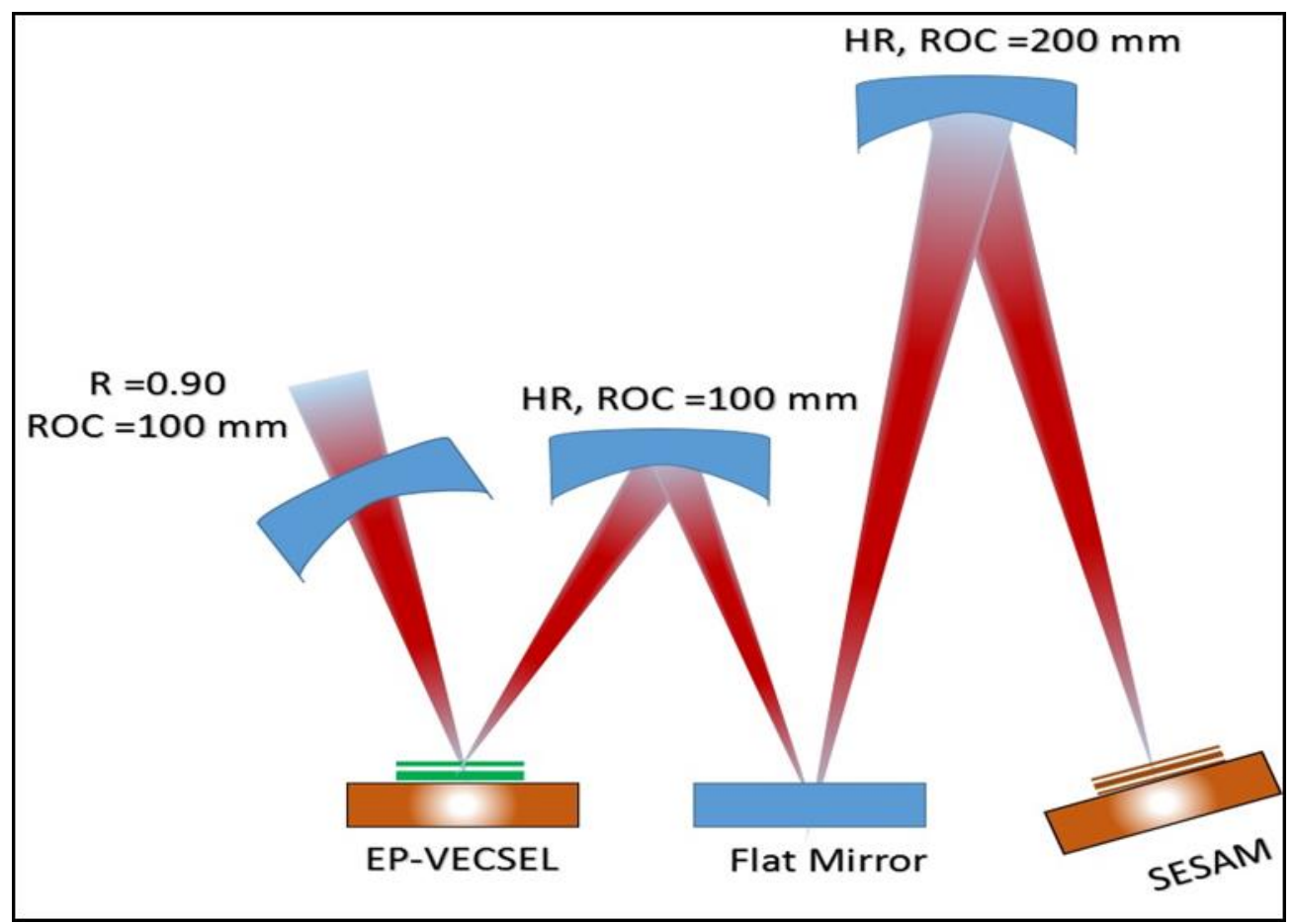

Figure 3. A schematic diagram of optical cavity layout for $980 \mathrm{~nm}$ mode-locked EP-VECSEL.

\section{RESULTS}

After the alignment, the laser was operating at $216 \mathrm{MHz}$ as indicated by radio frequency (RF) spectrum in Fig. 4. The spectrum was measured with resolution bandwidth of $10 \mathrm{kHz}$. The inset in Fig.4 shows a number of RF harmonics indicating stable mode-locked operation. The average output power of $8 \mathrm{~mW}$ was achieved with direct pump current value of $730 \mathrm{~mA}$. Intensity autocorrelation was used to measure pulse duration of $24 \mathrm{ps}$ (Fig. 5). The output spectrum of the sample was centered around $980 \mathrm{~nm}$ with the FWHM $=0.07 \mathrm{~nm}$ and is shown in the inset of Fig. 5. The pulses were 1.66 times transform-limited. The peak power was calculated to be $1.56 \mathrm{~W}$.

Slightly improved average output power could be achieved in the shorter cavity configuration. The output power was measured to be $34 \mathrm{~mW}$ with pulse duration of $42 \mathrm{ps}$ at a repetition rate of $499 \mathrm{MHz}$. Beam quality parameter $\mathrm{M}^{2}$ was measured to be $\sim 1.23$ in this configuration. We believe that the increase in output power in the $499 \mathrm{MHz}$ repetition rate setup was a result of more efficient energy storage in the laser upper-state and reduction of losses in shorter cavity. Furthermore, the pulse energy required to saturate the absorber should be constant, which implies a higher repetition rate should correspond to a higher average output power. 


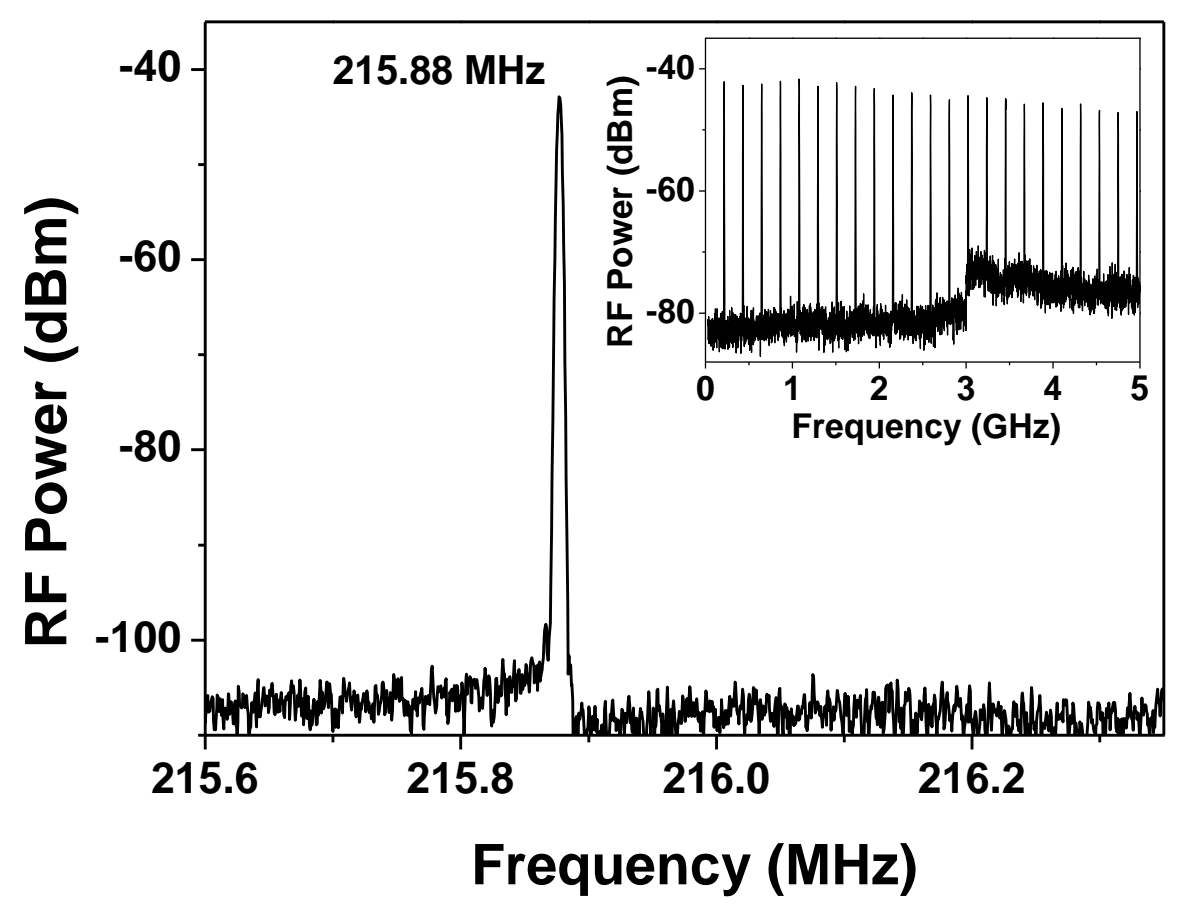

Figure 4. RF spectrum of mode-locked EP-VECSEL. The inset shows a number of subsequent harmonics of the spectrum.

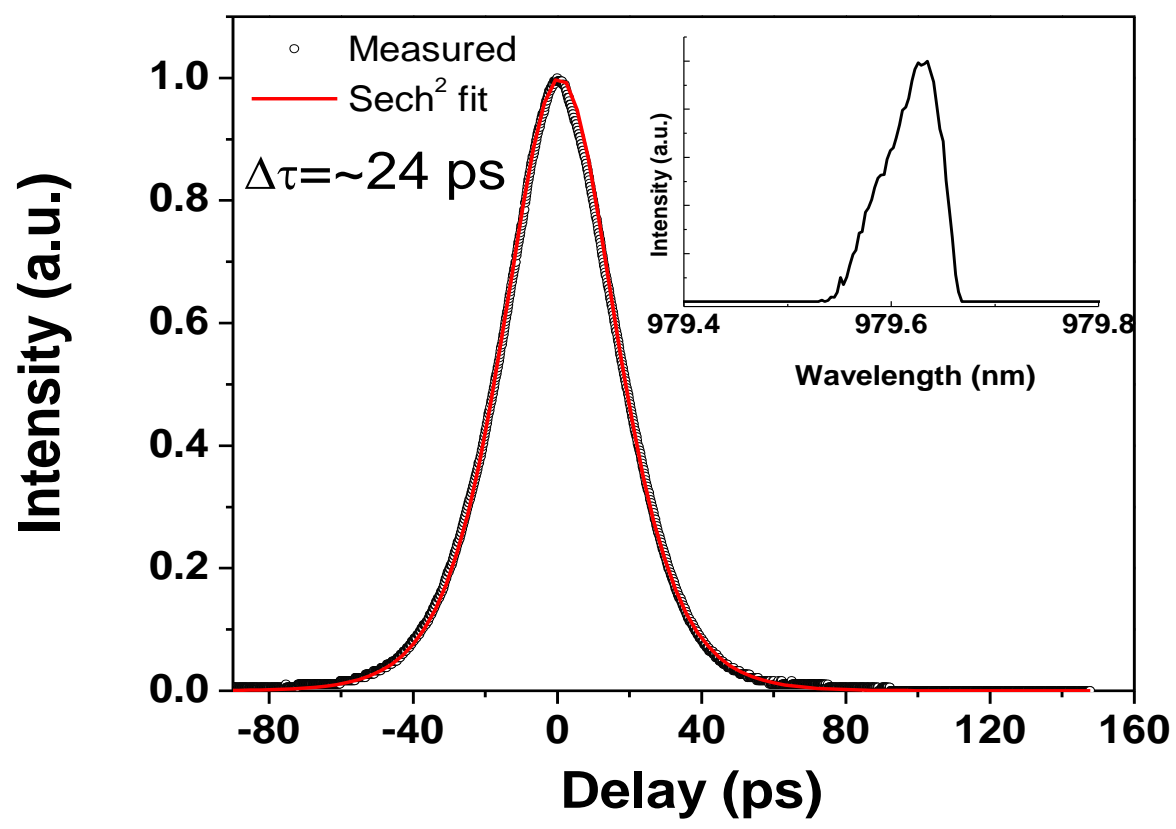

Figure 5. Autocorrelation trace of mode-locked EP-VECSEL indicating 24 ps pulse duration. The inset shows optical spectrum centred at $979.6 \mathrm{~nm}$. 
The $216 \mathrm{MHz}$ repetition rate was the minimum that could be achieved in our experiment. Operation in longer cavity would become unstable or would switch to harmonic mode-locking. Although OP-VECSELs were mode-locked in similar and lower repetition rate, it is typically considered hardly achievable as cavity round-trip time becomes significantly longer than upper state lifetime in the gain. Recently it was proposed that phase amplitude effect could be playing a role in addition to classical mode-locking image of interplay between gain and saturable absorber, especially were structures based on QW and QD are integrated providing different alpha factors. Deeper understanding of VECSEL dynamics is thus necessary to explore the lower limit of repetition rate in this type of lasers.

In summary, quantum dot saturable absorber was used to passively mode-lock EP- VECSEL at record low repetition rate of $216 \mathrm{MHz}$. The laser produced $8 \mathrm{~mW}$ average output power with $24 \mathrm{ps}$ pulse width at $980 \mathrm{~nm}$ emission wavelength. Peak power of $1.56 \mathrm{~W}$ was achieved. Further scaling of average output power and pulse duration will increase the peak power of EP-VECSEL to $>10 \mathrm{~W}$ level.

\section{ACKNOWLEDGEMENT}

We would like to thank Dr. Aram Mooradian for kindly providing EP-VECSELs samples for this work. Ali Alhazime also thanks Taibah University in Saudi Arabia for financial support.

\section{REFERENCES}

[1] B. Heinen, T. L. Wang, M. Sparenberg, A. Weber, B. Kunert, J. Hader, S. W. Koch, J. V. Moloney, M. Koch, and W. Stolz, Electronics Letters 48, 516-U102 (2012).

[2] M. Scheller, T. L. Wang, B. Kunert, W. Stolz, S. W. Koch, and J. V. Moloney, Electronics Letters 48, 588U123 (2012).

[3] M. Butkus, E.A. Viktorov, T. Erneux, C. J. Hamilton, G. Maker, G.P.A. Malcolm and E.U. Rafailov, accepted to Optics Express (2013).

[4] L. Kornaszewski, G. Maker, G. Malcolm, M. Butkus, E. U. Rafailov, and C. J. Hamilton, Laser \& Photonics Reviews 6, L20-L23 (2012).

[5] R. Aviles-Espinosa, G. Filippidis, C. Hamilton, G. Malcolm, K. J. Weingarten, T. Sudmeyer, Y. Barbarin, U. Keller, S. Santos, D. Artigas, and P. Loza-Alvarez, Biomedical Optics Express 2, 739-747 (2011).

[6] J. G. McInerney, A. Mooradian, A. Lewis, A. Shchegrov, E. M. Strzelecka, D. Lee, J. P. Watson, A. Liebman, G. P. Carey, B. D. Cantos, W. R. Hitchens, and D. Heald, Electronics Letters 39, 523-525 (2003).

[7] J. R. Orchard, D. T. Childs, L. C. Lin, B. J. Stevens, D. M. Williams, and R. A. Hogg, Japanese Journal of Applied Physics 50, 04DG05 (2011).

[8] K. Jasim, Q. Zhang, A. V. Nurmikko, E. Ippen, A. Mooradian, G. Carey, and W. Ha, Electronics Letters 40, $34-$ 36 (2004).

[9] K. Jasim, Q. Zhang, A. V. Nurmikko, A. Mooradian, G. Carey, W. Ha, and E. Ippen, Electronics Letters 39, 373-375 (2003).

[10]E. U. Rafailov, W. Sibbett, A. Mooradian, J. G. McInerney, H. Karlsson, S. Wang, and F. Laurell, Opt. Lett. 28, 2091-2093 (2003).

[11] A. A. Lagatsky, C. G. Leburn, C. T. A. Brown, W. Sibbett, S. A. Zolotovskaya, and E. U. Rafailov, Progress in Quantum Electronics 34, 1-45 (2010).

[12] K. G. Wilcox, M. Butkus, I. Farrer, D. A. Ritchie, A. Tropper, and E. U. Rafailov, Applied Physics Letters 94, 251105-251105-3 (2009).

[13] A. Lagatsky, F. Bain, C. Brown, W. Sibbett, D. Livshits, G. Erbert, and E. Rafailov, Applied Physics Letters 91, 231111-231111-3 (2007). 